\title{
Low-temperature renormalization group study of uniformly frustrated models for type-II superconductors
}

\author{
Giancarlo Jug \\ Max-Planck Institut für Physik komplexer Systeme, Außenstelle Stuttgart, Heisenbergstr. 1, \\ Postfach 800665 D-70569 Stuttgart (Germany) \\ Boris N. Shalaev \\ A.F.Ioffe Physical \& Technical Institute, Russian Academy of Sciences, \\ 194021 St.Petersburg (Russia) **.* \\ and Max-Planck Institut für Physik komplexer Systeme, D-70569 Stuttgart (Germany)
}

\begin{abstract}
We study phase transitions in uniformly frustrated $S U(N)$-symmetric $(2+\epsilon)$ dimensional lattice models describing type-II superconductors near the upper critical magnetic field $H_{c 2}(T)$. The low-temperature renormalization-group approach is employed for calculating the beta-function $\beta(T, f)$ with $f$ an arbitrary rational magnetic frustration. The phase-boundary line $H_{c 2}(T)$ is the ultraviolet-stable fixed point found from the equation $\beta(T, f)=0$, the corresponding critical exponents being identical to those of the non-frustrated continuum system. The critical properties of the $S U(N)$-symmetric complex Ginzburg-Landau (GL) model are then examined in $(4+\epsilon)$ dimensions. The possibility of a continuous phase transition into the mixed state in such a model is suggested.
\end{abstract}

PACS numbers: 74.60Ge, 74.60Ec 


\section{INTRODUCTION}

The challenging problem of the critical behavior of a type-II superconductor near the upper critical magnetic field $H_{c 2}(T)$ has a rich and long history going back to the seminal paper by Eilenberger [1] (see also [2]). It has been recognized for some time that an external magnetic field drastically changes the critical properties of superconductors. The magnetic field hinders the growth of the thermal fluctuations in the plane perpendicular to $\mathbf{H}$, since the growth of their correlation length is restricted by the magnetic length scale $\ell=\sqrt{h c / e H}$ which is much shorter than the coherence length $\xi$. This effect of dimensional reduction results in an enhancement of the longitudinal fluctuations leading, in particular, to the increase of the lower critical dimension from 2 to 4 .

If critical fluctuations are ignored, the uniform frustration (in the language of spin models of the vortex lattice) eventually leads to a continuous phase transition into the Abrikosov flux lattice state. On the one hand, in contrast to mean-field theory, the standard renormalization group (RG) approach in $6-\epsilon$ dimensions, in fact, failed so far to yield insight on the nature of the phase transition due to the appearence of an infinite number of invariant charges (relevant scaling variables) inherent to the non-renormalizable scalar $\phi^{4}$ field theory in a field [3]. Physical arguments (presented e.g. in [2]) support the existence of a first-order melting transition for the flux lattice. On the other hand, however, the conventional $1 / N$ expansion, when applied to the $S U(N)$-symmetric Ginzburg-Landau (GL) model with an $N$-component order parameter, gives a second-order phase transition above four dimensions [4 6]. The question of the nature of the phase transition from the normal into the mixed state of a type-II superconductor remains therefore an open problem, even before the effects of impurities and the topical question of the vortex glass are to be considered. In this paper we wish to investigate what is to some extent a new question, namely the nature of this phase transition when a periodic lattice potential is coupled to the superconducting order parameter.

It was shown some time ago [7,8] that the interaction between thermal fluctuations and the underlying crystal lattice can restore the phase transition into the mixed state. It is well known that the vortex lattice state has two broken symmetries: i) phase coherence, that is off-diagonal long-range order (ODLRO), and ii) translational symmetry. Including the interaction with an underlying lattice means that the translational symmetry is explicitly broken and lattice effects (for instance Harper's broadening and splitting of the Landau levels suppressing infinite degeneracy of the energy spectrum) become particular acute.

Lattice models of superconductors were introduced long ago and are of great interest by their own accord, being employed to study phase transitions in uniformly frustrated $\mathrm{XY}$ models [9], or in some artificial condensed matter structures like two-dimensional (2D) Josephson-junction arrays subject to a magnetic field [10] or in opals filled with a semiconductor or a metal [11.

Naturally, according to a naive point of view, near criticality the correlation length $\xi$ diverges and the system should "forget" about the discretness of the underlying lattice; however, this turns out not to be the case. In fact, in the continuum limit some specific uniformly- frustrated lattice models have been shown not to be equivalent to the conventional GL model [9]. If lattice effects are taken into account, in the long-wavelength limit 
one obtains an infinite set of effective (renormalizable) GL Hamiltonians corresponding to different rational values of the frustration $f=\Phi / \Phi_{0}$, where $\Phi=B a^{2}$ is the magnetic flux per plaquette and $\Phi_{0}=h c / 2 e$ the elementary flux quantum [5,9].

The main goal of the present paper is to consider the critical behavior of lattice models of type-II superconductors in the normal phase above $H_{c 2}(T)$. We shall apply the standard lowtemperature RG approach that was first introduced and developed in [12,13]. The method was first exploited for studying critical phenomena in superconductors (with $B=0$ ) in [14. We will show how the presence of a lattice induces a second-order phase transition in dimensions $d=2+\epsilon$, by giving explicitly the phase boundary in the low-temperature limit.

The remainder of this paper is organized as follows. In Section II the critical behavior of the $(2+\epsilon)$-dimensional $S U(N)$-symmetric lattice model is considered by means of the lowtemperature RG approach. Section III deals with the standard GL model in $4+\epsilon$ dimensions, therefore in the absence of the underlying lattice, and we show that the phase transition appears to be again of second order, although it remains impossible to reach a conclusion about the nature of the phase transition in $d=3$. Section IV contains a discussion of our results and of related issues in which lattice effects may play an important role and some concluding remarks.

\section{VORTEX-LATTICE PHASE TRANSITION IN $2+\epsilon$ DIMENSIONS}

\section{A. Effective action and low-temperature expansion}

We begin by considering the classical Hamiltonian of the $S U(N)$-symmetric nonlinear sigma model, defined on a square lattice with periodic boundary conditions:

$$
H=-J \sum_{<i, j>}\left|S_{i}^{a} \exp \left(i A_{i j}\right)-S_{j}^{a}\right|^{2}
$$

This is indeed the lattice version of the GL Hamiltonian, $S_{i}^{a}$ being a $N$-component complex unit vector with the constraint $S_{i}^{a} S_{i}^{a *}=1, a=1,2, \ldots, N$ and $J$ is a coupling constant. Here $\langle\cdots\rangle$ indicates that the summation is over all nearest-neighboring sites, as usual. $A_{i j}$ is a bond angle such that the sum around a plaquette is given by

$$
\sum_{\text {plaquette }} A_{i j}=2 \pi f
$$

with $f=p / q$ the so-called frustration; $p$ and $q$ are here mutually prime integers and $A_{i j}$ is defined by

$$
A_{i j}=\frac{2 \pi}{\Phi_{0}} \int_{i}^{j} d x_{\mu} A_{\mu}
$$

where $A_{\mu}=(-B y, 0,0)$ is the vector potential of the uniform magnetic field $B$ along the $z$ axis. From eq.(2.2) it follows that the magnetic flux through a plaquette is assumed to be a rational fraction of the magnetic flux quantum $\Phi_{0}$.

Consider the partition function associated with eq.(2.1), namely 


$$
Z=\int \prod_{i} d^{N} S_{i} d^{N} S_{i}^{*} \exp \left\{-\frac{H}{T}\right\} \delta\left(S_{i}^{a} S_{i}^{a *}-1\right)
$$

Notice that the Hamiltonian in eq.(2.1) has both a global nonabelian $S U(N)$ symmetry and a local $U(1)$ one whilst the fixed-length constraint imposed on the local spins is $0(2 N)$ symmetric.

The main steps in our calculations are as follows. To carry out the weak-coupling expansion for the theory given by eq.(2.1) a formal procedure based on integrating out the high-frequency components of the local spins $S_{i}^{a}$ will be applied. We shall make use of the following parametrization of spin variables [15]

$$
\begin{aligned}
S_{k}^{b} & =\pi_{k}^{b} \quad b=1,2, \ldots, N-1 \\
\pi_{k}^{2} & =\pi_{k}^{b} \pi_{k}^{b *} \\
S_{k}^{N} & =\sqrt{1-\pi_{k}^{2}} \exp \left(i \phi_{k}\right)
\end{aligned}
$$

where $\pi_{k}^{b}$ are small and slowly-varying fluctuations about the $N$ th-component's direction. Substituting the representation eq.(2.5) into eq.(2.4) and integrating out the modulus $\left|S_{k}^{N}\right|$, one arrives at the Lagrangian

$$
\begin{aligned}
Z & =\int \prod_{i} d^{N-1} \pi_{i} d^{N-1} \pi_{i}^{*} \exp \{-H / T\} \\
H & =H_{0}+H_{i n t} \\
H_{0} & =-J \sum_{<i, j>}\left|\pi_{i}^{b} \exp \left(i A_{i j}\right)-\pi_{j}^{b}\right|^{2} \\
H_{\text {int }} & =-J \sum_{<i, j>}\left|\sqrt{1-\pi_{i}^{2}} \exp \left(\phi_{i}-\phi_{j}+A_{i j}\right)-\sqrt{1-\pi_{j}^{2}}\right|^{2}
\end{aligned}
$$

Here the Jacobian factor equals unity. The advantages of this parametrization are quite evident because if $T \rightarrow 0$ the spin-wave fields $\pi_{i}^{b}$ can be treated as free lattice fields. As these fields fluctuate, a contribution to the effective action for the phases $\phi_{i}$ will arise. At the same time, the field $\phi(i)$ does not react back onto $\pi_{i}^{b}$ in the renormalization procedure.

The calculations in the one-loop approximation may be readily carried out by means of the standard momentum-shell recursion relation technique developed in 16] (see, also [15,17]). To produce a systematic low-temperature perturbation theory one has to expand nonlinearities such as $\sqrt{1-\pi_{i}^{2}}$ in $H_{\text {int }}$ in powers of $\pi_{i}^{2}$ and to integrate out short-wavelength degrees of freedom. Let us decompose the Fourier-transformed spin field according to

$$
\begin{array}{llrl}
\pi^{b}(q) & =\pi_{<}^{b}(q)+\pi_{>}^{b}(q) & \\
\pi^{b}(q) & =\pi_{<}^{b}(q) & & 0<q<\Lambda^{\prime} \\
\pi^{b}(q) & =\pi_{>}^{b}(q) & & \Lambda^{\prime}<q<\Lambda
\end{array}
$$

with the purpose of integrating out the short-wavelength fields $\pi_{>}^{b}(q)$. Here $\Lambda$ and $\Lambda^{\prime}$ are momentum cutoffs and $q$ stands here for the reciprocal lattice coordinates. Calculations in the lowest order of perturbation theory yield 


$$
\frac{H_{i n t}}{T}=\frac{2}{T} \sum_{<i, j>} \cos \left(\phi_{i}-\phi_{j}+A_{i j}\right)\left(1-\frac{1}{2}<\pi_{i}^{2}>-\frac{1}{2}<\pi_{j}^{2}>\right)
$$

where the angular brackets $\langle\cdots\rangle$ stand for the straight Gaussian integration over the modes $\pi_{>}(q)$, and $J$ is absorbed in $T$. From eq.(2.8) the temperature renormalization is easily seen to be

$$
\frac{1}{T^{\prime}}=\frac{1}{T}\left(1-<\pi_{i}^{2}>\right)
$$

Setting the magnetic field (or, equivalently, the frustration) to zero one may easily take the long-distance limit of the model and find the bare propagator for the $\pi_{>}^{b}(q)$ fields, namely

$$
G_{a b}(q)=<\pi_{>}^{a}(q) \pi_{>}^{b}(-q)>=\frac{T}{q^{2}} \delta_{a b}
$$

The most divergent part of the Goldstone propagator behaves like $1 / q^{2}$ for small $q$, giving rise to the dominant logarithmic term in $\left\langle\pi_{i}^{2}\right\rangle=(N-1) \ln \frac{\Lambda}{\Lambda^{\prime}}$. Carrying out the trivial calculations as described in detail in [15], one is lead to the familiar expression for the one-loop beta function

$$
\beta(T)=(d-2) T-\frac{(N-1) T^{2}}{\pi}
$$

The problem now is to extend this approach to the uniformly frustrated model of eq.(2.1), that is when the magnetic field is switched back on.

\section{B. Lattice frustrated models}

Since we are, in this paper, interested in the critical behavior of the lattice frustrated model, we shall first of all note some properties of the Azbel-Harper-Hofstadter operator $\hat{L}$ 18 21 describing a 2D Bloch charged particle subject to a uniform magnetic field. Here $\hat{L}$ is the inverse of the quadratic part of the action $H_{0}$ which one may formaly regard as the electron hopping Hamiltonian. Using a compact notation, the Hamiltonian $H_{0}$ in eq.(2.6) can be rewritten as

$$
H_{0}=\sum_{n} \pi_{n}^{b *} \hat{L} \pi_{n}^{b}
$$

where by definition (due to the choice of the most conventional Landau gauge) $\hat{L}$ acts on the Bloch wave function in the following way

$$
\begin{aligned}
\hat{L} \pi^{b}(n) & =\exp \left(i k_{x}\right) \pi_{k}^{b}(n-1)+\exp \left(-i k_{x}\right) \pi_{k}^{b}(n+1) \\
& +2 \cos \left(k_{y}+2 \pi \frac{n p}{q}\right) \pi_{k}^{b}(n) \\
\pi^{b}(n) & =\exp (i k n) \pi_{k}^{b}(n)
\end{aligned}
$$

where $n=1, \ldots, q$ is a coordinate in the magnetic cell and the wave vector $k$ ranges over the reduced Brillouin zone: $-\pi / a<k_{x}<\pi / a,-\pi / q a<k_{y}<\pi / q a$. It is worth noting that if $n$ 
labels the components of a $q$-component "vector" $\pi_{k}^{b}(n)$, then the operator $\hat{L}$ acts as a $q \times q$ Hermitean matrix. In the case of $q=4$ one has, for example

$$
\hat{L}=\left(\begin{array}{llll}
2 \cos \left(k_{x}\right) & \exp \left(-i k_{y}\right) & 0 & \exp \left(i k_{y}\right) \\
\exp \left(i k_{y}\right) & 2 \cos \left(k_{x}+2 \pi f\right) & \exp \left(-i k_{y}\right) & 0 \\
0 & \exp \left(i k_{y}\right) & 2 \cos \left(k_{x}+4 \pi f\right) & \exp \left(-i k_{y}\right) \\
\exp \left(-i k_{y}\right) & 0 & \exp \left(i k_{y}\right) & 2 \cos \left(k_{x}+6 \pi f\right)
\end{array}\right)
$$

Therefore, this Hamiltonian $H_{0}$ can be thought of as that corresponding to a particle hopping along $q$ sites around a ring [22, 23]. The spectrum of $H_{0}$ is known to posess a finite set of $q$ magnetic sub-bands, each state in these sub-bands being $q$-fold degenerate instead of the infinite degenerancy inherent to the continuous problem 23.

The magnetic translational symmetry properties result from the local gauge invariance of the lattice theory, eq.(2.1). This implies that under the translation $\mathbf{r} \rightarrow \mathbf{r}+\mathbf{a}$ the propagator in eq. (2.10) transforms as follows

$$
G_{a b}\left(\mathbf{r}+\mathbf{a}, \mathbf{r}^{\prime}+\mathbf{a}\right)=\exp \left\{\frac{i \pi}{\Phi_{0}}[\mathbf{B} \times \mathbf{a}]\left(\mathbf{r}-\mathbf{r}^{\prime}\right)\right\} G_{a b}\left(\mathbf{r}, \mathbf{r}^{\prime}\right)
$$

From eq.(2.15) it follows that $G_{a b}\left(\mathbf{r}, \mathbf{r}^{\prime}\right)$ at coinciding points does not depend on $\mathbf{r}$. On the other hand, for the propagator from eq.(2.15) one has the resolvent spectral decomposition

$$
G_{a b}\left(\mathbf{r}, \mathbf{r}^{\prime}\right)=\delta_{a b} \sum_{n=1}^{q} \sum_{\alpha=1}^{q} \sum_{\mathbf{k}} E_{n \mathbf{k}}^{-1} \Psi_{n \mathbf{k} \alpha}(\mathbf{r}) \Psi_{n \mathbf{k} \alpha}^{*}\left(\mathbf{r}^{\prime}\right)
$$

where $n=1, \ldots, q$ labels the magnetic band number, and $\Psi_{n \mathbf{k} \alpha}(\mathbf{r})$ are eigenfunctions of $\hat{L}$ defined in the magnetic Brillouin zone, $-\pi / a<k_{x}<\pi / a$ and $-\pi / q a<k_{y}<\pi / q a$, forming the basis of the $q$-dimensional irreducible projective representation of the magnetic translation group. The exact energy spectrum $E_{n \mathbf{k}}$ does not depend on the quantum number $\alpha=1, \ldots, q$.

There exists a remarkable property of the energy spectrum resulting from the local gauge invariance of the lattice theory, eq.(2.1). From this local gauge invariance it follows that $E_{n \mathbf{k}}$ depends on $\mathbf{k}$ only through the parameter $\Delta=2-\cos \left(q k_{x}\right)-\cos \left(q k_{y}\right)$ and of course on $q$ [24]

$$
E_{n \mathbf{k}}=E_{n}(\Delta, q)
$$

From the point of view of the critical properties of the nonlinear $\sigma$-model under consideration, one can see that only states lying near the bottom of the lowest magnetic sub-band $(n=1)$ are relevant. Here it works in a similar way, as in the well-known lowest Landau level (LLL) projection approximation for the continuous theory.

Within the effective mass approximation the energy spectrum as a function of a quasimomentum near the band's bottom $(\Delta=0)$ reads

$$
E_{1 \mathbf{k}}=e_{0}(q)+\frac{e_{2}(q)}{2}\left\{\left(q k_{x}\right)^{2}+\left(q k_{y}\right)^{2}\right\}
$$


with $e_{0,2}(q)$ being coefficients of the Taylor expansion of $E_{1 \mathbf{k}}$ in powers of $\mathbf{k}$. Here $e_{2}(q)^{-1}$ is proportional to an effective mass in the $(x, y)$-plane, $e_{0}(q)$ being proportional to a chemical potential. It is here rather essential that the effective mass and chemical potential are some functions of $q$.

In order to find $\beta(T)$ we have to calculate $\left\langle\pi_{i}^{2}>\right.$, which is given by

$$
\begin{aligned}
<\pi_{i}^{2}> & =\sum_{a=1}^{q} G_{a a}(\mathbf{r}, \mathbf{r}) \\
& =q \sum_{n=1}^{q} \sum_{\mathbf{k}} E_{n \mathbf{k}}^{-1}
\end{aligned}
$$

The factor $q$ appears as a result of the degeneracy of the spectrum and summation over $\alpha$. Making use the effective mass approximation for the lowest magnetic sub-band, and evaluating the integral over $\mathbf{k}$ in eq.(2.19), we arrive at the following expression

$$
\begin{aligned}
<\pi_{i}^{2}> & =q \sum_{\mathbf{k}} \frac{2 e_{2}(q)^{-1}}{\left(q k_{x}\right)^{2}+\left(q k_{y}\right)^{2}+m_{0}^{2}} \\
& =\frac{2(N-1) q}{e_{2}(q)} \ln \frac{\Lambda}{\Lambda^{\prime}} .
\end{aligned}
$$

Here, $m_{0}^{2}=2 e_{0}(q) / e_{2}(q)$ denotes some effective mass. With the help of eq.(2.9) and of eq.(2.20), one can readily derive the beta-function in the one-loop approximation

$$
\beta(T, q)=(d-2) T-\frac{2(N-1) q T^{2}}{\pi e_{2}(q)}
$$

This expression is the lattice version of eq.(2.11). It leads to the nontrivial ultraviolet-stable fixed point located at

$$
T^{*}(q)=\frac{(d-2) \pi e_{2}(q)}{2(N-1) q}
$$

We see that, in fact, eq. (2.22) gives the phase transition line $H_{c 2}(T)$ of the lattice model, eq.(2.1). The peculiarity of the beta-function, eq.(2.21), lies in its explicit dependence on the frustration $q$ revealing a multifractal type structure. This follows from the properties of the spectrum of a single-electron in a magnetic field and a periodic potential (leading to the so-called "Hofstadter's butterfly" multifractal structure [19,20]). From the physical point of view this peculiarity looks quite natural since the phase transition temperature must depend on the applied magnetic field. After setting $q=1$, or $H=0, e_{2}(q)$ becomes $e_{2}(1)=2$ and the function $\beta(T)$ coincides with eq.(2.9).

The critical exponent for the correlation length $\nu$ follows immediately by differentiating eq. (2.21) at the fixed point. Keeping only the leading term in $\epsilon$ one obtains

$$
\nu=-\frac{1}{\beta^{\prime}\left(T^{*}\right)}=\frac{1}{\epsilon}
$$


To determine Fisher's critical exponent $\eta$ one may apply the conventional momentum-shell $\mathrm{RG}$ approach to first order in $\epsilon$ as described in detail in [16,17]. After carrying out the standard RG computational procedure, we are led to the following expression

$$
\eta(T)=2-d+\frac{(2 N-1) q T}{\pi e_{2}(q)}
$$

Note that eq.(2.22) and eq.(2.24) yield the usual one-loop result $\eta=\frac{\epsilon}{2(N-1)}$.

We have seen above that the $(2+\epsilon)$-dimensional frustrated lattice model undergoes a continuous phase transition on the coexistance curve $H_{c 2}(T)$ and is characterized by universal critical exponents which we have calculated. A physical interpretation of this conclusion is quite simple: the infinite degenerancy inherent to a charged particle moving in a uniform magnetic field is lifted by a commensurate potential suppressing the dimensional reduction effect and favouring the flux lattice state [6]. At least at the lowest order in $\epsilon$ the frustration $q$ drops out of the critical exponents, these being identical to those for the conventional $0(2 N)$ symmetric Heisenberg ferromagnet. For $N=1$ the expressions obtained above obviously

show a singularity in the factor $\frac{1}{N-1}$, which reflects the special properties of the neutral $2 \mathrm{D}$ XY model.

\section{WEAK-COUPLING EXPANSION FOR THE GINZBURG-LANDAU MODEL}

The approach which has been developed until now may be extended to the continuous GL model subject to a uniform magnetic field $\mathbf{B}$ in $d=4+\epsilon$ dimensions, in the spirit of the work of Lawrie and Athorne [14,25]. This model is described by the Hamiltonian

$$
H=\int d^{d} x\left|\left(\partial_{\mu}+i \frac{2 \pi}{\Phi_{0}} A_{\mu}\right) \Psi_{a}\right|^{2}
$$

where the summations over $\mu=1, \ldots, d$ and $a=1, . ., N$ are understood in eq.(3.1). Here $\Psi=\left[\Psi_{1}, \ldots, \Psi_{N}\right]$ is an $N$-component complex order parameter, the fixed-length constraint $|\Psi|^{2}=1$ being imposed on the local fields. The vector potential $A_{\mu}$ is now taken within the symmetric gauge

$$
\mathbf{A}=\frac{1}{2} \mathbf{B} \times \mathbf{r}
$$

where $\mathbf{B}$ is taken along the $z$ axis. The partition function associated with eq.(3.1) reads

$$
Z=\int \prod_{a=1}^{N} D \Psi_{a} D \Psi_{a}^{*} \exp \left(-\frac{H}{T}\right) \delta\left(|\Psi|^{2}-1\right)
$$

We shall make use of the same parametrization of $\Psi_{a}$ exploited in Section II

$$
\begin{aligned}
\Psi_{b}(\mathbf{r}) & =\pi_{b}(\mathbf{r}) \quad b=1,2, \ldots, N-1 \\
\pi^{2}(\mathbf{r}) & =\pi_{b}(\mathbf{r}) \pi_{b}^{*}(\mathbf{r}) \\
\Psi_{N}(\mathbf{r}) & =\sqrt{1-\pi^{2}(\mathbf{r})} \exp (i \phi(\mathbf{r}))
\end{aligned}
$$


Inserting eq. (3.4) into eq.(3.3) and integrating out the $\left|\Psi_{N}\right|$, we obtain the effective Hamiltonian convenient for generating the low-temperature expansion

$$
H=\int d^{d} x\left[\left|\left(\partial_{\mu}+i \frac{2 \pi}{\Phi_{0}} A_{\mu}\right) \pi_{b}\right|^{2}+\left(\partial_{\mu} \phi+\frac{2 \pi}{\Phi_{0}} A_{\mu}\right)^{2}+\left(\partial_{\mu} \sqrt{1-\pi^{2}}\right)^{2}\right]
$$

Observe that no Jacobian arises from the elimination of $\left|\Psi_{N}\right|$. To determine the temperature renormalization in the lowest order of perturbation theory, we have to take into account only the first two relevant terms in eq.(3.4) and disregard the third one describing the interaction between the transverse degrees of freedom $\pi_{b}$. The RG equation obtained in this way is

$$
\frac{1}{T^{\prime}}=\frac{1}{T}\left(1-<\pi^{2}(\mathbf{r})>_{s}\right)
$$

where $\langle\cdots\rangle_{s}$ stands for integrating over the short-wavelength transverse fields $\pi_{b}$.

Notice that the non gauge-invariant correlation function

$$
G_{a b}\left(\mathbf{r}, \mathbf{r}^{\prime}\right)=<\pi_{a}(\mathbf{r}) \pi_{b}\left(\mathbf{r}^{\prime}\right)>
$$

is the Green function of the $d$-dimensional Schrodinger operator

$$
\left(-i \partial_{\mu}-\frac{2 \pi}{\Phi_{0}} A_{\mu}\right)^{2} G_{a b}\left(\mathbf{r}, \mathbf{r}^{\prime}\right)=\delta_{a b} \delta\left(\mathbf{r}-\mathbf{r}^{\prime}\right)
$$

The exact solution of eq.(3.8) in an arbitrary gauge reads [26]

$$
\begin{aligned}
G_{a b}\left(\mathbf{r}, \mathbf{r}^{\prime}\right) & =\delta_{a b} \exp \left(-i \frac{2 \pi}{\Phi_{0}} \int_{\mathbf{r}}^{\mathbf{r}^{\prime}} d x_{\mu} A_{\mu}\right)(4 \pi)^{\frac{2-d}{2}} \int_{0}^{\infty} d u \frac{u^{\frac{2-d}{2}}}{2 \sinh (u \omega / 2)} \\
& \times \exp \left\{-\frac{\left(z-z^{\prime}\right)^{2}}{4 u}-\frac{\omega}{8} \operatorname{coth}\left[\frac{1}{2} u \omega\left(\left(x-x^{\prime}\right)^{2}+\left(y-y^{\prime}\right)^{2}\right)\right]\right\}
\end{aligned}
$$

where $\omega=\frac{2 e B}{c}$ is the cyclotron frequency (here we have set $\hbar=1$ and $2 m=1$ ) and $z$ and $z^{\prime}$ are $(d-2)$-dimensional longitudinal coordinates. The integral in eq.(3.9) is taken over the straight line connecting the points $\mathbf{r}$ and $\mathbf{r}^{\prime}$.

Near the phase boundary $H_{c 2}(T)$, only the lowest Landau level gives a dominant contribution to the gauge-invariant quantity $G(\mathbf{r}, \mathbf{r})$ entering eq.(3.6). The LLL approximation leads to a nice simplification of eq.(3.9)

$$
\begin{aligned}
G_{a b}\left(\mathbf{r}, \mathbf{r}^{\prime}\right) & =\delta_{a b} \exp \left\{-i \frac{2 \pi}{\Phi_{0}} \int_{\mathbf{r}}^{\mathbf{r}^{\prime}} d x_{\mu} A_{\mu}-\frac{\omega}{8}\left[\left(x-x^{\prime}\right)^{2}+\left(y-y^{\prime}\right)^{2}\right]\right\} \\
& \times(4 \pi)^{-\frac{d}{2}} \int_{0}^{\infty} d u u^{\frac{2-d}{2}} \exp \left\{-\frac{u \omega}{2}-\frac{\left(z-z^{\prime}\right)^{2}}{4 u}\right\}
\end{aligned}
$$

In going from eq.(3.9) to eq.(3.10) we have made the following substitution in eq.(3.9): $\operatorname{coth}(u \omega / 2) \rightarrow 1$ and $\sinh (u \omega / 2) \rightarrow \frac{1}{2} \exp (u \omega / 2)$ (justified by the limit $B \rightarrow \infty$ of the LLL approximation).

To make further calculations more transparent, we will make use of the mixed coordinatemomentum representation for $G_{a b}\left(\mathbf{r}, \mathbf{r}^{\prime}\right)$. After carrying out the Fourier transformation in the longitudinal variables $z, z^{\prime}$ eq.(3.10) becomes 


$$
G_{a b}\left(x, x^{\prime}, y, y^{\prime}, \mathbf{k}\right)=\delta_{a b} \exp \left\{-i \frac{2 \pi}{\Phi_{0}} \int_{\mathbf{r}}^{\mathbf{r}^{\prime}} d x_{\mu} A_{\mu}-\frac{\omega}{8}\left[\left(x-x^{\prime}\right)^{2}+\left(y-y^{\prime}\right)^{2}\right]\right\} \frac{\omega}{k^{2}+\xi^{2}}
$$

where $\mathbf{k}$ is a $d-2$-dimensional vector and $\xi$ is a correlation length in the longitudinal directions. The important conclusion one may draw from eq.(3.11) is that a dimensional reduction effect takes place in the physics of our model, eq.(3.1). We have also set an irrelevant factor to unity.

With the result of eq.(3.11) at hand, we can now readily evaluate $\left\langle\pi^{2}(\mathbf{r})\right\rangle_{s}$

$$
<\pi^{2}(\mathbf{r})>_{s}=\frac{(N-1) \omega}{\pi} \ln \frac{\Lambda}{\Lambda^{\prime}}
$$

Eq.(3.6) and eq.(3.12) yield the beta-function in the one-loop approximation as well as the phase boundary, namely

$$
\begin{aligned}
\beta(T, B) & =(d-4) T-\frac{(N-1) \omega}{\pi} T^{2} \\
T^{*}(B) & =\frac{\pi(d-4)}{(N-1) \omega}
\end{aligned}
$$

It is remarkable that the calculation of the critical exponents, like the one carried out in Section II, leads now to the same universal values as given by eq.(2.22) and eq.(2.24), independently of $B$.

We have seen that in contrast to the results obtained e.g. in [3] for the $(6-\epsilon)$-dimensional case, a continuous phase transition occurs in the GL model in $4+\epsilon$ dimensions. It is described by the critical exponents of the $0(2 N)$-symmetric Heisenberg ferromagnet (in the one-loop approximation). From the field-theoretical point of view we are now dealing with the dimensional reduction effect within the Abelian Higgs model defined by an Euclidean Lagrangian, eq.(3.1) (or, equivalently, in the $N$-component scalar QED), in a large external magnetic field. The analogous effect was recently shown to occur also in the conventional $4 D$ spinor QED subject to an extremely high magnetic field like in a vicinity of a neutron star [27.

\section{CONCLUSIONS}

It has been shown that the $(2+\epsilon)$-dimensional $S U(N)$-symmetric uniformly-frustrated lattice spin model undergoes a second-order phase transition, described by the universal critical exponents of the $0(2 N)$-symmetric Heisenberg ferromagnet irrespectively of the value of the frustration. Our calculations provide no evidence of a first-order phase transition. This result was found to hold both for the $(2+\epsilon)$-dimensional lattice model as well as for the $(4+\epsilon)$-dimensional continuos GL model in an applied magnetic field. The occurence of the second-order phase transition is in this case in contrast to the RG calculations carried out in $(6-\epsilon)$-dimensions [3]. Contrary to the conventional RG approach based on the $\phi^{4}$-theory [9], the low-temperature RG approach employed in this paper works for an arbitrary rational value of the frustration $f=\Phi / \Phi_{0}$. It allows us to compute the phase transition boundary line $H_{c 2}(T)$, which is a very difficult calculation problem within the standard $\phi^{4}$-theory. In 
the case of the lattice model the coexistence curve exhibits a very complicated structure (in fact, reminiscent of the "devil's staircase" [9] inherited from the Hofstadter's butterfly's structure [19.20]) which can be determined only numerically for all integer values of $q$.

Our treatment can be easily extended to the large- $N$ limit. But the situation here is somewhat unclear since different groups of researchers have come to different conclusions concerning the nature of the transition. On the one hand, our results - being in good agreement with those obtained within the $\frac{1}{N}$-expansion by Radzihovsky [6] and by the authors of [5] - give evidence in favour of a continuous phase transition. In particular, the non-trivial fixed point responsible for the second-order phase transition was found. Moreover, from the equation for the correlation length $\xi$ it follows that there is a divergence of $\xi$ at crticality. One the other hand, the calculation of the effective potential within the $\frac{1}{N}$ expansion as presented in [4] , gives evidence for a first-order phase transition. The origin of this discrepancy in conclusions is rather subtle and probably lies in non-commutativity of the large- $N$ limit and the thermodynamic limit (see, for a discussion, ref. [6]).

The main problem still open for future research is how to extend the results obtained by means of the low-temperature RG approach to the $N=1$ case. Thus, we have seen that the underlying lattice plays an essential important role near the upper critical magnetic field $H_{c 2}(T)$ restoring (like weak disorder) the continuos phase transition into the fluxlattice state. This is indeed the main result of our paper and we have commented in the Introduction on possible direct applications of the model we have studied.

\section{ACKNOWLEDGEMENTS}

This work was supported by the Russian Foundation for Basic Research Grant No. 9602-16858, the NATO Collaborative Research Grant No. OUTR.CRG960838 and by the EC contract No. ERB4001GT957255. The authors are most grateful to the Max-PlanckInstitute für Physik komplexer Systeme, Außenstelle Stuttgart, where a considerable part of this work was carried out, for kind hospitality and the use of its facilities. One of the authors (BNS) is also most grateful to the Department of High Energy Physics of the International School for Advanced Studies in Trieste, where part of this work was carried out, for support and warm hospitality. He is deeply grateful to S.Shenoy and R.Iengo for interesting discussions and valuble comments. It is also a great pleasure to thank H.Capellmann, G.Eliashberg, A.Nersesyan, A.Protogenov and W.Selke for helpful discussions. 


\section{REFERENCES}

* On leave of absence from: INFM and Istituto di Scienze Matematiche, Fisiche e Chimiche, Università di Milano a Como, Via Lucini 3, 22100 Como (Italy) (permanent address)

** Permanent address

[1] Eilenberger G. Phys.Rev. 164, 628 (1967)

[2] For useful reviews, see M.Rasolt and Z.Tesanovich, Rev.Mod.Phys. 64, 709 (1992); G.Blatter, M.V.Feigel'man, V.B.Geshkenbein, A.I.Larkin, and V.M.Vinokur, Rev.Mod.Phys. 66, 1125 (1994)

[3] Brézin E., Nelson D.R., and Thiaville A. Phys.Rev. B 31, 7124 (1985); see also Newman T., and Moore M.A., Phys.Rev. B 54, 6661 (1996)

[4] Affleck I., and Brézin E., Nucl.Phys. B 257, [FS14], 451 (1985)

[5] S.A.Ktitorov, B.N.Shalaev, and L.Jastrabik, Phys.Rev. B 49, 15248 (1994)

[6] Radzihovsky L., Phys.Rev.Lett. 74 , 4722 (1995); see also the discussion by Herbut I.F., and Tesanovich Z., Phys.Rev.Lett. 76 , 4550 (1996), and Radzihovsky L., Phys.Rev.Lett. 76,4551 (1996)

[7] Toner J., Phys.Rev.Lett. 66, 2523 (1991)

[8] S.A.Ktitorov, Yu.V.Petrov, B.N.Shalaev, and V.S.Sherstinov, Int.J.Mod.Phys. B 6,1209 (1992)

[9] Choi M.Y., and Doniach S., Phys.Rev. B 31, 4516 (1985)

[10] Granato E., and Kosterlitz J.M.,Phys.Rev.Lett. 65, 1267 (1990)

[11] Bogomolov V.N., Kumzerov Yu.A., Romanov S.G., and Zhuravlev V.V., Physica C 208, 371 (1993)

[12] Migdal A.A., Sov.Phys. JETP 42, 413 (1975)

[13] Polyakov A.M., Phys.Lett. B 59, 79 (1975)

[14] Lawrie I.D., and Athorne C., J.Phys: Math.Gen. A 16, L587 (1983)

[15] Kogut J.B., Rev.Mod.Phys. 51, 659 (1979)

[16] Nelson D.R. and Pelcovits R.A., Phys.Rev. B 16, 2191 (1977)

[17] Brézin E. and Zinn-Justin J., Phys.Rev. B 14, 3110 (1976)

[18] Harper P.G., Proc.Phys.Soc.London A 68, 874 (1955)

[19] Azbel M.Ya., Sov.Phys.JETP 19, 634 (1964)

[20] Hofstadter D.R., Phys.Rev. B 14, 2239 (1976)

[21] Wen X.G., and Zee A., Nucl.Phys. B 316, 641 (1989)

[22] Wiegmann P.B., and Zabrodin A.V.,Nucl.Phys. B 422, [FS], 495 (1994)

[23] Zinn-Justin J., Quantum Field Theory and Critical Phenomena, Clarendon Press, Oxford (1993); Ma S.-K., Modern Theory of Critical Phenomena, W.A.Benjamin, Inc., (1976)

[24] Thouless D.J., Phys.Rev. B 28, 4272 (1983)

[25] Hikami S., Progr. Theor.Phys. 62, 226 (1979)

[26] Feynman R., and Hibbs A., Quantum Mechanics and Path Integrals McGraw Hill Book Company, New York (1965)

[27] Miransky V.A., Progr. Theor.Phys.Suppl. 123, 49 (1996) 